\title{
Complement-Activating Capacity of Autoantibodies Correlates With Disease Activity in Bullous Pemphigoid Patients
}

\section{OPEN ACCESS}

Edited by:

Philippe Saas,

INSERM U1098 Interactions

Hôte-Greffon-Tumeur and Ingénierie

Cellulaire et Génique, France

Reviewed by:

Ralf J. Ludwig,

Universität zu Lübeck, Germany

Yale Liu,

Second Affiliated Hospital of Xi'an

Jiaotong University, China

Takashi Hashimoto,

Osaka University, Japan

${ }^{*}$ Correspondence:

Cassian Sitaru

cassian@mail.sitaru.eu;

cassian.sitaru@

mail.medizin.uni-freiburg.de

†These authors have contributed equally to this work

Specialty section:

This article was submitted to

Inflammation,

a section of the journal

Frontiers in Immunology

Received: 16 July 2018

Accepted: 31 October 2018

Published: 20 November 2018

Citation:

Chiorean RM, Baican A, Mustafa MB,

Lischka A, Leucuta D-C, Feldrihan V, Hertl M and Sitaru C (2018)

Complement-Activating Capacity of

Autoantibodies Correlates With

Disease Activity in Bullous Pemphigoid

Patients. Front. Immunol. 9:2687.

doi: 10.3389/fimmu.2018.02687

\begin{abstract}
Roxana M. Chiorean ${ }^{1,2 t}$, Adrian Baican ${ }^{1 \dagger}$, Mayson B. Mustafa ${ }^{1}$, Annette Lischka ${ }^{1}$, Daniel-Corneliu Leucuta ${ }^{3}$, Vasile Feldrihan ${ }^{4}$, Michael Hertl ${ }^{5}$ and Cassian Sitaru ${ }^{1,6 *}$
\end{abstract}

${ }^{1}$ Department of Dermatology, Medical Faculty, Medical Center - University of Freiburg, Freiburg, Germany, ${ }^{2}$ Department of Dermatology, University of Medicine and Pharmacy luliu Hatieganu, Cluj-Napoca, Romania, ${ }^{3}$ Department of Medical Informatics and Biostatistics, University of Medicine and Pharmacy luliu Hatieganu, Cluj-Napoca, Romania, ${ }^{4}$ Department of Immunology, University of Medicine and Pharmacy luliu Hatieganu, Cluj-Napoca, Romania, ${ }^{5}$ Department of Dermatology and Allergology, Philipps-University, Marburg, Germany, ${ }^{6}$ Centre for Biological Signaling Studies(BIOSS), University of Freiburg, Freiburg, Germany

Background: Bullous pemphigoid is a subepidermal blistering skin disease, associated with autoantibodies to hemidesmosomal proteins, complement activation at the dermal-epidermal junction, and dermal granulocyte infiltration. Clinical and experimental laboratory findings support conflicting hypotheses regarding the role of complement activation for the skin blistering induced by pemphigoid autoantibodies. In-depth studies on the pathogenic relevance of autoimmune complement activation in patients are largely lacking. Therefore, the aim of this study was to investigate the pathogenic relevance of complement activation in patients with bullous pemphigoid. Complement activation by autoantibodies in vivo as measured by the intensity of complement C3 deposits in the patients' skin and ex vivo by the complement-fixation assay in serum was correlated with the clinical disease activity, evaluated by Autoimmune Bullous Skin Disorder Intensity Score (ABSIS) and Bullous Pemphigoid Disease Area Index (BPDAI), as well as, with further immunopathological findings in patients with bullous pemphigoid.

Results: Complement-activation capacity of autoantibodies ex vivo, but not deposition of complement in the perilesional skin of patients, correlates with the extent of skin disease (measured by ABSIS and BPDAl) and with levels of autoantibodies.

Conclusions: Our study provides for the first time evidence in patients for a pathogenic role of complement activation in bullous pemphigoid and should greatly facilitate the development of novel diagnostic tools and of more specific therapies for complement-dependent autoimmune injury.

Keywords: bullous pemphigoid, complement-activating capacity of autoantibodies, complement-binding test, disease activity, ABSIS, BPDAI

\section{BACKGROUND}

Autoantibodies induce tissue damage in a series of autoimmune diseases, including rheumatoid arthritis, Goodpasture syndrome, myasthenia gravis, and autoimmune bullous dermatoses (1-8). Demonstration of the pathogenic potential of autoantibodies in these diseases greatly facilitated the development of effective B cell-targeted therapies (9). Binding of pathogenic autoantibodies to their 
targets induces tissue damage by various mechanisms. Autoantibodies may exert a direct effect just by binding to their target mediated by steric hindrance and/or by triggering the transduction of a signal to the cell. In other conditions, in addition to binding to their target antigen, autoantibodies need to interact with factors of the innate immune system, including the complement system and inflammatory cells in order to unfold their pathogenic potential (10). In patients with autoantibody-mediated diseases, deposits of complement components are frequently found in the affected tissues as an immunopathological marker of an inappropriate local complement activation (11, 12). Beyond their diagnostic significance, these clinical laboratory observations suggest a pathogenic role for complement activation in autoimmune diseases $(9,12)$.

The pathogenic significance of the complement activation in the setting of autoimmune injury has been extensively addressed using different ex vivo and animal models of autoimmune diseases (13-21). The results of these studies provide ample information suggesting that, in several conditions, including rheumatoid arthritis, myasthenia gravis, and epidermolysis bullosa acquisita, autoantibodies unfold their pathogenic potential by activating the complement cascade $(13,15,17)$. In other diseases such as pemphigus vulgaris or anti-laminin 332 pemphigoid, complement activation by autoantibodies appears to be an epiphenomenon and is not required for tissue injury $(16,22-24)$.However, studies using experimental models of pemphigoid diseases provided partly conflicting results on the role of complement activation (14, 18-20). A new and interesting potential therapeutic target is represented by the C5 fraction of complement. While C5aR1 showed a proinflammatory effect in a mouse model of bullous pemphigoid and epidermolysis bullosa acquisita, C5aR2 demonstrated an anti-inflammatory effect in the same mouse model of bullous pemphigoid $(21,25)$. Understanding the role and mechanisms of complement activation in autoimmune diseases provides a basis for developing more specific diagnostic assays and therapeutic approaches targeting key complement components and pathogenic events.

Bullous pemphigoid (BP) is a prototypical organ-specific autoimmune dermatosis associated with complement activation (26). Deposits of several complement components, of which C3 deposition is of significance for the routine diagnosis, are characteristically found in patients with bullous and gestational pemphigoid $(27,28)$. These findings are matched by observations in several animal models of bullous pemphigoid induced by the passive transfer of antibodies against BP180 demonstrating deposits of complement $\mathrm{C} 3$ at the dermal-epidermal junction of the diseased mice or hamsters $(18,19,29)$. The pathogenic significance of the complement activation has been addressed in vivo using mice deficient for several complement components

\footnotetext{
Abbreviations: ABSIS, Autoimmune Bullous Skin Disorder Intensity Score; BP, bullous pemphigoid; BPDAI, Bullous Pemphigoid Disease Area Index; BSA, bovine serum albumine; C3, complement component 3; ELISA, enzyme-linked immunosorbent assay; IF, immunofluorescence; IgG, immunoglobulin G; PBS, phosphate-buffered saline.
}

and pharmacological inhibition of complement activation using cobra venom factor $(14,18-20,30,31)$. In several earlier studies, activation of complement was shown to be a prerequisite for blister induction by autoantibodies. However, intriguing results of more recent studies challenged the major pathogenetic role of the complement system in pemphigoid diseases. Specifically, skin fragility was also demonstrated to be induced in a complement-independent manner. Collagen XVII was depleted in cultured normal human keratinocytes using antigen-specific rabbit polyclonal IgG. Also, skin fragility was induced in neonatal collagen XVII-humanized mice by passive transfer of $\mathrm{F}\left(\mathrm{ab}^{\prime}\right) 2$ fragments of IgG autoantibodies against collagen XVII from bullous pemphigoid patients or rabbit (19). Furthermore, passive transfer of autoantibodies from bullous pemphigoid patients was shown to induce blister formation in neonatal C3-deficient collagen XVII-humanized mice (20). A possible mechanism of depletion is represented by binding of IgG autoantibodies and internalization from the cell membrane (32).

Additional studies using ex vivo models of pemphigoid diseases revealed complex and partly conflicting results (3234), summarized in Figure 1. While these conflicting data may reflect different pathogenetic mechanisms of the autoantibodyinduced tissue injury in various models, controversy surrounds the pathogenic relevance of complement activation in patients.

Therefore, in the present study, we aimed to investigate the pathogenic significance of complement activation in pemphigoid patients. For this purpose, we have measured the complementbinding capacity of patients' autoantibodies in vivo and ex vivo and have assessed their correlation with the clinical disease activity, as well as, their capacity to induce dermal-epidermal separation ex vivo. The results of our study documented a correlation of the autoantibody complement-binding capacity with the extent of the blistering disease, strongly suggesting a pathogenic role of complement activation in pemphigoid patients.

\section{METHODS}

\section{Patients}

We have retrospectively analyzed 90 patients with $\mathrm{BP}$, from the Department of Dermatology, at the University of Medicine and Pharmacy "Iuliu Hatieganu," Cluj-Napoca, Romania. The study was approved by the local Ethic Committee and performed in accordance with the Declaration of Helsinki. Written informed consent was obtained from all patients, in adherence to the Helsinki Principles. Inclusion criteria for patients with BP were: (1) presence of skin bullae, (2) subepidermal blisters on histopathological examination and (3) linear deposits of IgG and/or C3 at the dermo-epidermal junction, detected by direct immunofluorescence. In addition, we have also evaluated the presence of anti-BP180 +/- anti-BP230 autoantibodies, measured by the enzyme-linked immunosorbent assay (ELISA) method and titers of IgG autoantibodies binding to the epidermal side, measured by indirect immunofluorescence (IF) microscopy on salt-split skin (4).

Epidemiological, clinical and immunological data were collected from patients' medical records. In addition to the age 


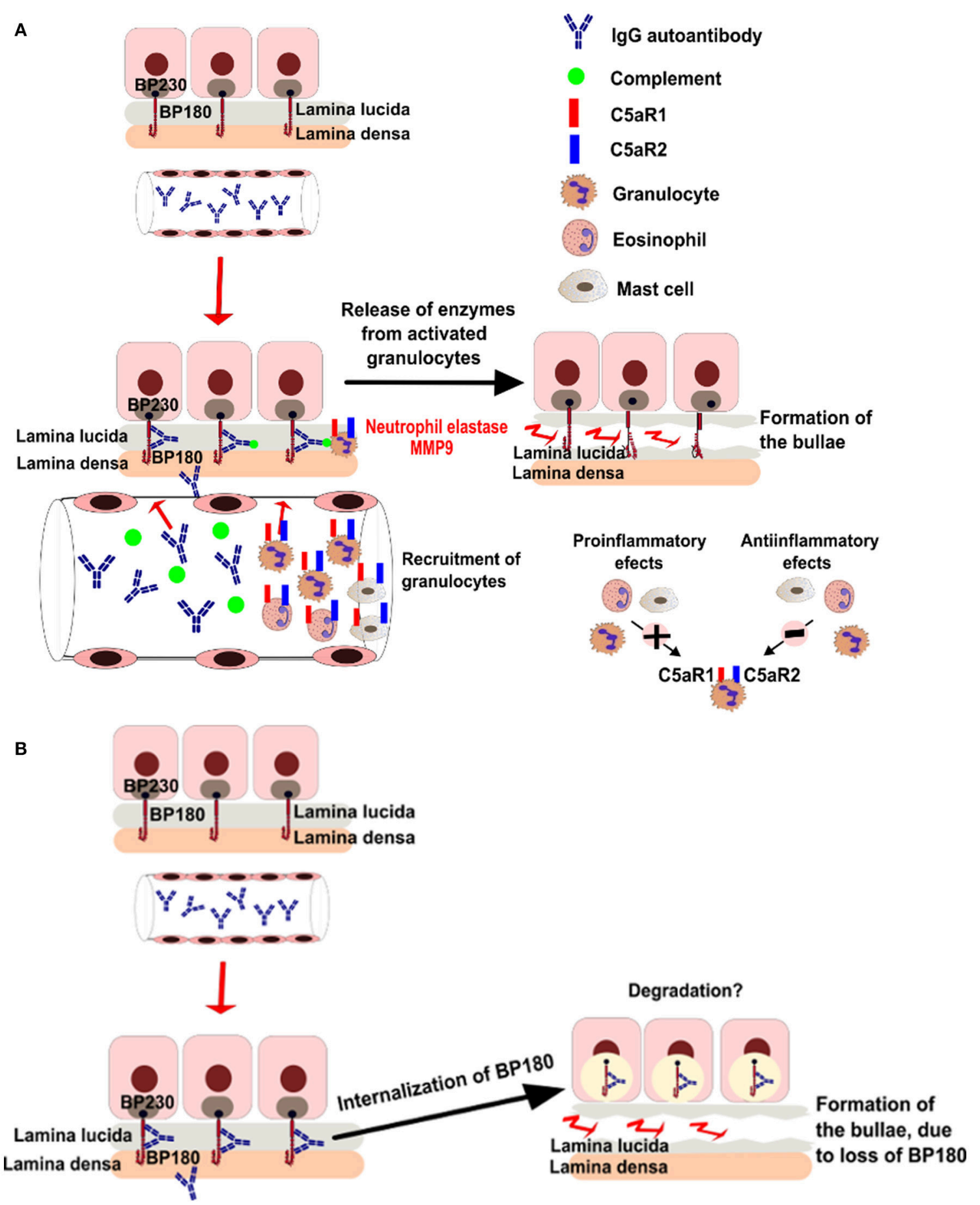

FIGURE 1 | Putative pathogenic mechanisms of blistering in bullous pemphigoid. (A) Complement dependent pathways of bullae formation. Binding of IgG autoantibodies at the dermal epidermal junction leads to complement activation and recruitment of neutrophils. Activated neutrophils release proteolytic enzymes, inducing dermal-epidermal separation. (B) Complement-independent pathways of bullae formation. Binding of lgG autoantibodies to BP180 leads to depletion of the protein by internalization.

of onset and gender, we collected further variables, including erythrocyte sedimentation rate (ESR) at diagnosis and history of neurological comorbidities (stroke, dementia, Parkinson, epilepsy). Female to male ratio was 1.19 (49:41). Eleven BP patients presented oral mucosa involvement. The objective cutaneous and mucosal components of Autoimmune Bullous 

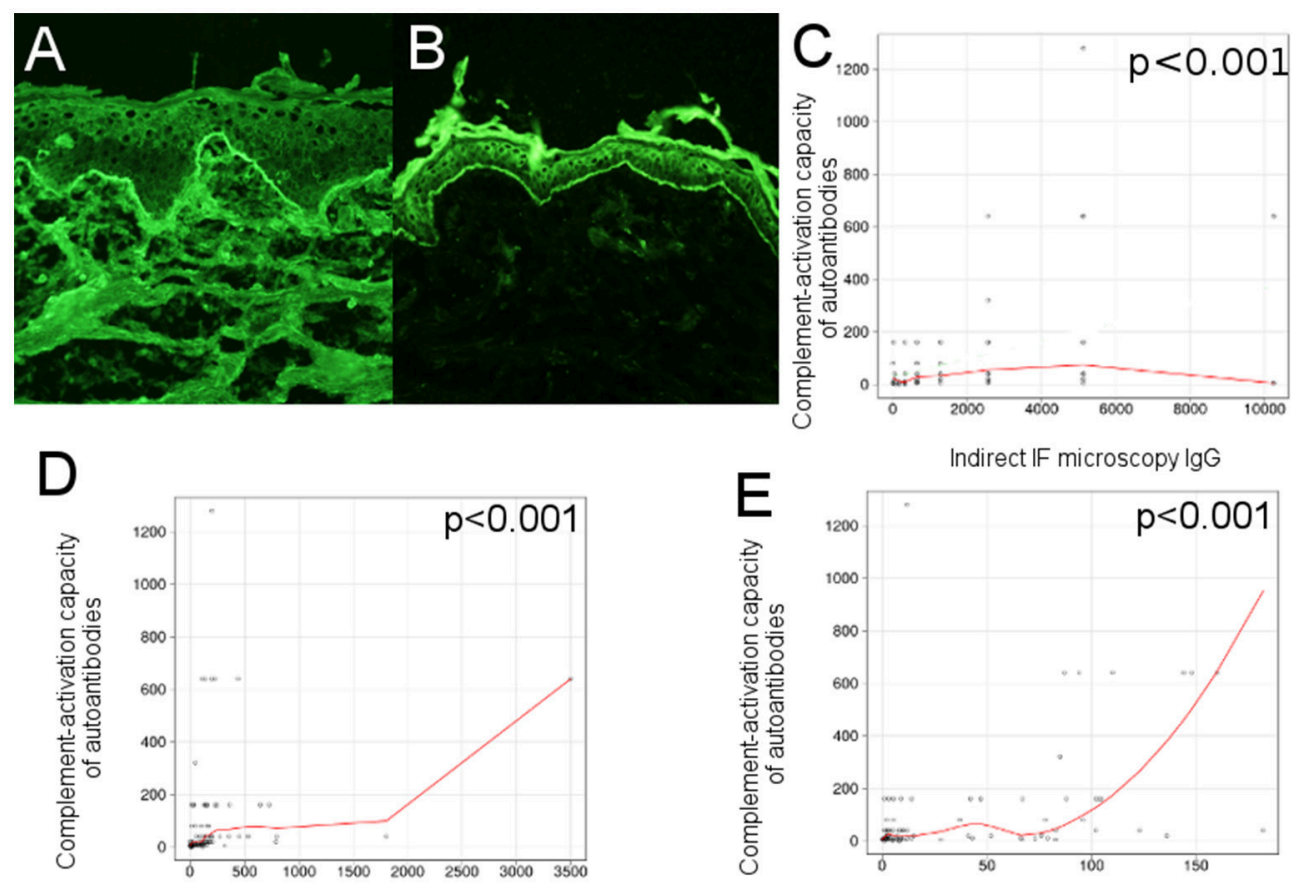

ELISA BP180 NC16A (U/ml)

ELISA BP230(U/ml)

FIGURE 2 | Complement-activation potential of circulating lgG anti-basement membrane zone autoantibodies correlates with their levels in bullous pemphigoid patients. (A) Indirect immunofluorescence microscopy on normal human foreskin shows binding of IgG autoantibodies from the serum of a BP patient at the dermal-epidermal junction. (B) Complement-binding test shows linear C3 deposits along the basement membrane of normal human foreskin, when using serum from a BP patient, demonstrating activation of complement by lgG autoantibodies. Relation of ex vivo complement-activating capacity of autoantibodies and (C), titers of circulating IgG anti-basement membrane zone autoantibodies, measured by indirect IF microscopy; (D), titers of circulating IgG anti-BP180 NC16A, measured by ELISA; (E), titers of circulating IgG anti-BP230, measured by ELISA $(n=90)$. The red line represents the locally weighted scatterplot smoothing line.

Skin Disorder Intensity Score (ABSIS) and Bullous Pemphigoid Disease Area Index (BPDAI) scores have been evaluated using clinical pictures of BP patients $(35,36)$. ESR was assessed both as a continuous and dichotomial variable. For the dichotomial value, we used a cut-off of $30 \mathrm{~mm} / \mathrm{h}$, as this was proved to represent a risk factor for lethal outcome in patients with BP (37).

The assessed immunopathological parameters were the direct and indirect IF microscopy findings, complement-binding test results, extent of dermal-epidermal split and titers of anti-BP180 and anti-BP230 autoantibodies.

\section{Direct Immunofluorescence}

Sections from perilesional patients' skin were fixed for $10 \mathrm{~min}$ in acetone and then washed 2 times in phosphate buffered saline (PBS). In a further step, they were incubated with rabbit antihuman IgG-FITC (Dako) diluted 1:100 in PBS and rabbit-antihuman C3-FITC (Dako) diluted 1:100 in PBS. Sections were washed 2 times, $10 \mathrm{~min}$, in $\mathrm{PBS}$ ( $\mathrm{pH} \mathrm{7.2).} \mathrm{At} \mathrm{the} \mathrm{end,} \mathrm{sections}$ were mounted in $50 \%$ glycerol-PBS and analyzed by fluorescence microscopy. The sections were analyzed using an Olympus BX40 microscope and pictures from representative areas were acquired. The images were saved in.tiff or.jpeg format.

\section{Indirect Immunofluorescence Microscopy}

Neonatal human foreskin, obtained from routine circumcision, was washed in cold PBS, cut into pieces of $6 / 15 \mu \mathrm{m}$, embedded in optimum cutting temperature compound and stored at $-20^{\circ} \mathrm{C}$ until use. Sections were washed 2 times in PBS ( $\mathrm{pH} \mathrm{7.2),} \mathrm{then}$ incubated in a humid chamber, at room temperature, in two steps $(1 \mathrm{~h}$ each). In the first step, the sections were incubated with serial dilutions of sera from BP patients and controls in $1 \%$ BSA PBS. The second step involved incubation with goat antihuman IgG $(\mathrm{H}+\mathrm{L})$, (AF488) diluted 1:100 in PBS (1\% BSA). After each step, sections were washed 3 times, 10 min, in PBS ( $\mathrm{pH}$ 7.2). At the end, sections were mounted in 50\% glycerol-PBS and analyzed by fluorescence microscopy. An example is illustrated in Figures 2A, 3A. We have used the end-point titration of sera by indirect immunofluorescence microscopy as a robust indicator of autoantibody reactivity in patients with bullous pemphigoid.

\section{Measurement of the \\ Complement-Activating Capacity of Autoantibodies ex vivo by Complement-Binding Test Method}

The patient sera have been frozen at $-80^{\circ} \mathrm{C}$ and then thawed and kept at 4C. The complement binding test 

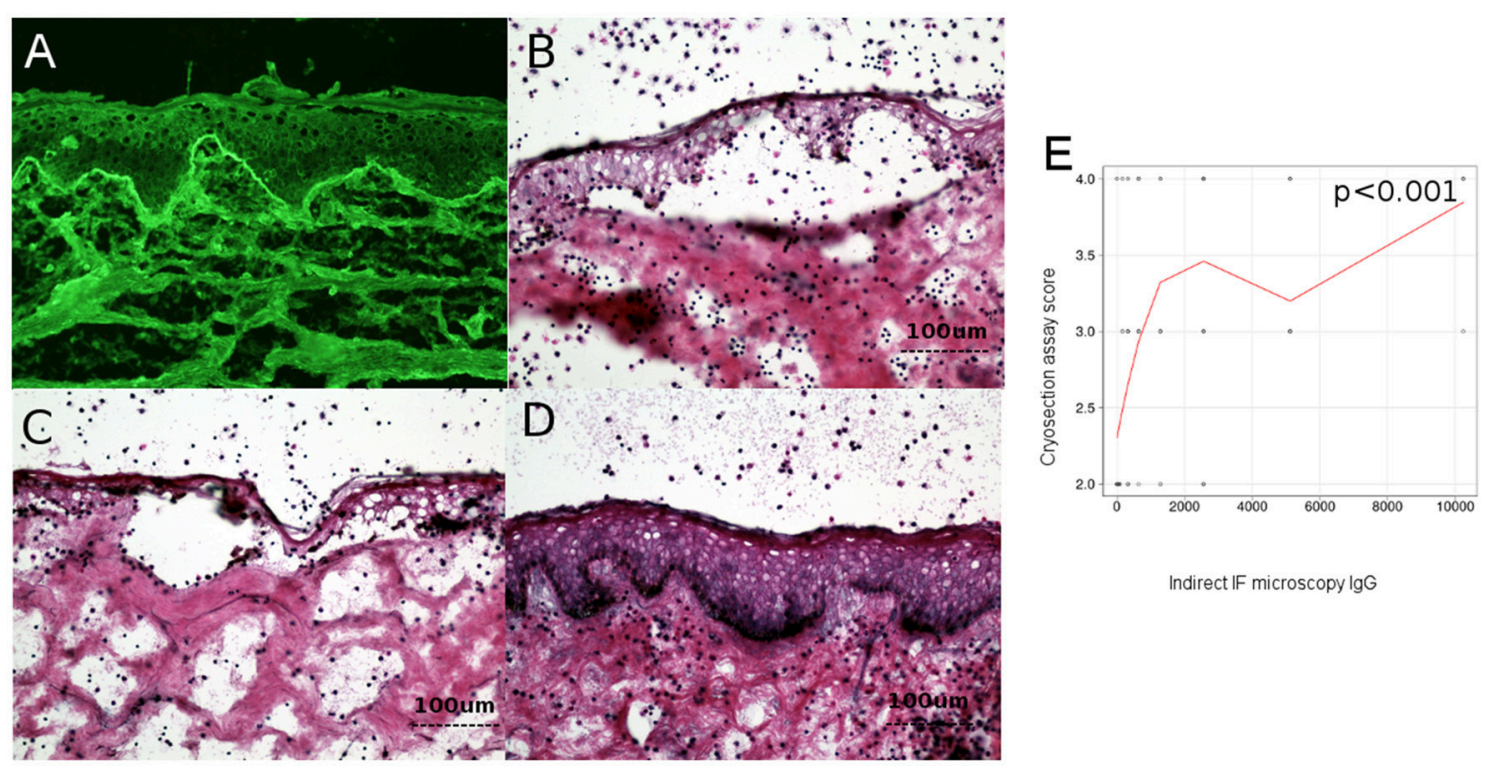

Indirect IF microscopy lgG

FIGURE 3 | Level of circulating lgG anti-basement membrane zone autoantibodies correlates with dermo-epidermal separation in patients with bullous pemphigoid. (A) Indirect immunofluorescence microscopy on normal human foreskin, using BP patient sera. (B,C) Cryosection assay ex vivo of normal human foreskin, incubated with a BP serum pool (B) and BP serum from a patient (C). Subsequent addition of normal human leukocytes leads to recruitment of neutrophils at the dermal-epidermal junction. Activation of neutrophils is induced by BP serum, leading to dermal-epidermal split. (D) Cryosection assay ex vivo of normal human foreskin, incubated with serum from healthy donors. Subsequent addition of normal human leukocytes does not lead to recruitment or activation of neutrophils at the dermal-epidermal junction and does not induce dermal-epidermal split. (E) Relation of titers of circulating lgG anti-basement membrane zone autoantibodies with dermal-epidermal separation in patients with bullous pemphigoid $(n=90)$. The red line represents the locally weighted scatterplot smoothing line.

was performed after the patients' sera were incubated at $56^{\circ} \mathrm{C}$ for $30 \mathrm{~min}$ to inactivate the complement components.

Neonatal human foreskin was prepared in the same manner as described above. Sections were washed 2 times in PBS ( $\mathrm{pH}$ 7.2 ), then incubated in a humid chamber, at room temperature, in three steps ( $1 \mathrm{~h}$ each). In the first step, the sections were incubated with serial dilutions of sera from BP patients and controls, in PBS. In a second step, incubation was made with normal human fresh serum, as a source of complement (stored at $-80^{\circ} \mathrm{C}$ before use), diluted at 1:5 in gelatin veronal buffer (Gelatin Veronal Buffer, BostonBio, Fisher Scientifics; Cat.no: IBB-290). The third step involved incubation with goat antiserum to human C3 (Polyclonal goat antibody to human C3-FITC, MPBiomedicals), diluted 1:100 in PBS ( $\mathrm{pH}$ 7.2). After each step, sections were washed 3 times, $10 \mathrm{~min}$, in PBS ( $\mathrm{pH} 7.2$ ). At the end, sections were mounted in 50\% glycerol-PBS and analyzed by fluorescence microscopy.

An example is illustrated in Figure 2B.

\section{Detection of BP180- and BP230-Specific Autoantibodies}

Levels of autoantibodies against the hemidesmosomal antigens BP180 and BP230 were measured using commercially available immunoassays (MBL laboratories, Nagoya, Japan), according to the manufacturer's instructions $(38,39)$.

\section{Ex vivo Model of Antibody-Induced Granulocyte-Dependent Dermal-Epidermal Separation}

The ability of BP patients' autoantibodies to activate human leukocytes was evaluated using an ex vivo assay of antibodyinduced granulocyte-dependent dermal-epidermal separation in cryosections of normal human skin, as described previously (34). Each experiment using the cryosection assay was performed twice to insure reproducibility of the results. The extent of dermal-epidermal separation (split) was assessed by two different observers and scored on a scale from 1 to 4 , as follows: level 0-no split; level 1-split present in $<25 \%$ of the basement membrane, but in more than 3 distinct places; level 2-split between 25 and $50 \%$ of the basement membrane; level 3 -split between 50 and $75 \%$ of the basement membrane; level 4 -split on $>75 \%$ of the basement membrane.

Examples of cryosection assay are illustrated in Figures 3B-D.

\section{Measurement of Complement Activation in Patients' Skin}

Direct IF microscopy pictures were taken with a 20x objective, using a Olympus BX40 microscope. The fluorescence intensity was assessed by two independent blinded observers using Image J software, version $1.47 \mathrm{v}$. The fluorescence intensity at the basement membrane was analyzed by choosing 50 points (using Multipoint selections tool), while background fluorescence was evaluated by selecting the whole dermis 
area, (using Freehand selections tool), as shown in detail in Figure 7. As we have seen a moderate agreement of intraand inter- observer measurements, we have decided to further perform statistical analysis, using the results obtained after evaluation of one picture for each patient. A mean value of the fluorescence intensities was calculated as follows: fluorescence at the basement membrane mean $=$ (fluorescence at the basement membrane observer $1+$ fluorescence at the basement membrane observer 2)/2; Fluorescence of the background mean $=$ (Fluorescence of the background observer $1+$ fluorescence of the background observer 2)/2. Then, the following values were calculated: fluorescence difference $=$ (fluorescence at the basement membrane mean-fluorescence of the background mean) and fluorescence ratio $=$ (fluorescence at the basement membrane mean/fluorescence of the background mean). Further, we have performed a correlation of fluorescence difference and fluorescence ratio with the complement-activation capacity by autoantibodies.

\section{Statistical Analysis}

Association between continuous variables was assessed using the Spearman correlation. The Mann Whitney U test, medians and interquartile range were used for non-normally distributed variables. The relation between dichotomial variables was assessed using contingency tables with absolute values and percentages and Fisher's exact test. For all tests, a two-tailed $p$ value $<0.05$ was considered statistically significant. Statistical analysis was performed using the $\mathrm{R}$ software environment for statistical computing and graphics, version 2.12.1 (copyright $\mathrm{R}$ Development Core Team, 2010) (40).

\section{RESULTS}

In order to compute the sample size for one of the main objectives of the study we considered as target effect size a minimum interesting Spearman correlation coefficient value of 0.3 . For the simulation we considered the use of an exact test for Spearman correlation coefficient, with $80 \%$ power to detect the effect size, a 0.05 level of significance, and a two-tail $p$-value. The simulated sample size value obtained in these circumstances was 82 subjects (GPower 3.0.10 software, Germany).

\section{Complement-Activation Potential of Circulating IgG Anti-Basement Membrane Zone Autoantibodies Correlates With Their Levels in Bullous Pemphigoid Sera}

In an initial evaluation of the complement-activating capacity of autoantibodies, we correlated the level of circulating IgG antibasement membrane zone autoantibodies, measured by indirect IF microscopy and ELISA with titers of the complement-binding test. Complement activation correlated with levels of circulating IgG anti-basement membrane zone autoantibodies measured by indirect IF microscopy ( $r=0.41$ [95\% CI 0.19-0.68], $p<0.001)$ (Figure 2C), level of anti-BP180 NC16A autoantibodies $(r=0.55$
[95\% CI 0.03-0.56], $p<0.001$; Figure 2D) and level of antiBP230 autoantibodies, $(r=0.46$ [95\% CI 0.17-0.7], $p<0.001$; Figure 2E), both measured by ELISA.

We have observed a correlation between the level of circulating IgG autoantibodies, measured by indirect IF microscopy and the level of anti-BP180-NC16A autoantibodies $(r=0.22$ [95\% CI -0.02 to 0.34$], p=0.04)$ and BP230-specific autoantibodies ( $r=0.33$ [95\% CI 0.25-0.64], $p=0.001)$, as measured by ELISA.

\section{Level of Circulating IgG Anti-Basement Membrane Zone Autoantibodies, but Not Complement Activation by Autoantibodies ex vivo Correlates With Dermo-Epidermal Separation in Patients With Bullous Pemphigoid}

To investigate the pathogenic relevance of complement activation ex vivo, measured by complement binding test, we have analyzed the correlation between the level of circulating IgG anti-basement membrane zone autoantibodies measured by indirect IF microscopy and ELISA and the complementactivation capacity of autoantibodies-as measured by the complement-binding test, with the extent of dermo-epidermal separation level in cryosection assay. Dermo-epidermal separation level in cryosection assay correlated with level of IgG autoantibodies measured by indirect IF $(r=0.4$ [95\% CI $0.2-0.5], p<0.001$ ) (Figure 3E), but not complement activation by autoantibodies, as measured by the complement-binding test $(p=0.09)$.

\section{Level of Circulating BP180 NC16A-, but Not BP230-Specific Autoantibodies Correlates With Disease Activity in Patients With Bullous Pemphigoid}

We observed a correlation between the level of circulating IgG anti-basement membrane zone autoantibodies measured by indirect IF microscopy and the ABSIS score of cutaneous involvement $(r=0.25$ [95\% CI -0.06 to 0.51 ], $p=0.03$; Figure 4A), BPDAI global score ( $r=0.29$ [ $95 \% \mathrm{CI}-0.04$ to 0.52 ], $p=0.01$ ) (Figure 4D), BPDAI score of total skin involvement $(r$ $=0.29$ [95\% CI 0-0.54], $p=0.01$ ) and BPDAI score of blistering activity ( $r=0.31$ [95\% CI -0.01 to 0.55$], p=0.006$ ), but not BPDAI score of urticaria/erythema $(p=0.17)$.

We did not find a statistically significant association between the serum levels of IgG anti-basement membrane zone autoantibodies and the ABSIS objective oral score $(p=0.28)$ and BPDAI mucosal score $(p=0.28)$. Further, we did not observe a statistically significant difference between the levels of circulating IgG autoantibodies, measured by indirect IF microscopy, in the patients with associated mucosal involvement vs. patients with only cutaneous involvement $(p=0.32)$.

In patients with positive ELISA values, we observed a statistically significant correlation between the level of circulating BP180 NC16A-specific autoantibodies measured by ELISA and the ABSIS score of cutaneous involvement $(r=0.63$ [95\% CI 


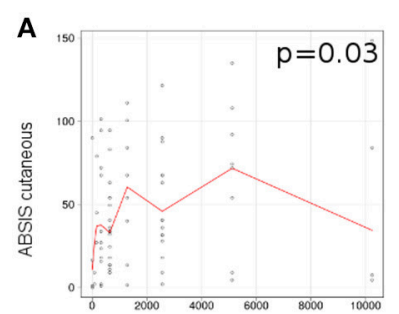

Indirect IF microscopy lgG

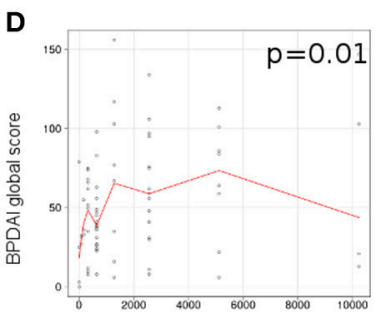

Indirect IF microscopy IgG

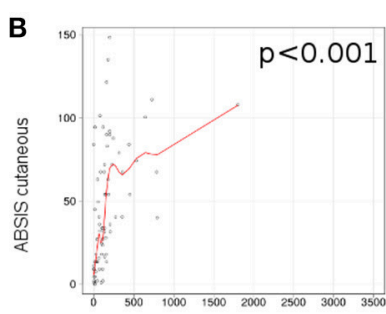

ELISA BP180 NC16A (U/ml)

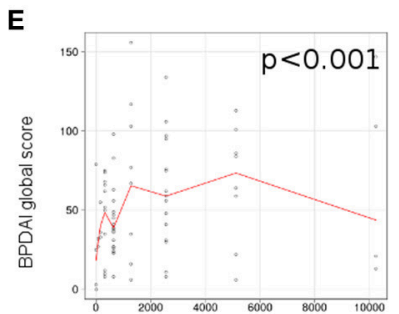

ELISABP180 NC16A (U/ml)

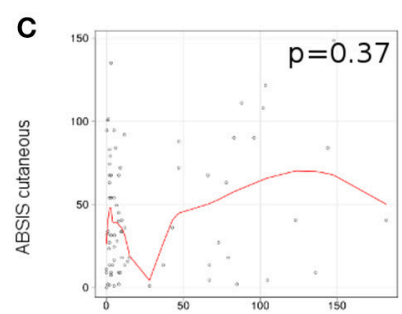

ELISA BP230 (U/ml)

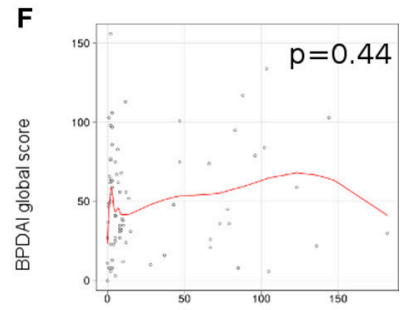

$\operatorname{ELISA} B P 230(\mathrm{U} / \mathrm{ml})$

FIGURE 4 | Level of circulating anti-BP180 NC16A, but not anti-BP230 autoantibodies, measured by ELISA correlates with disease activity in patients with bullous pemphigoid. Relation of titers of circulating IgG anti-basement membrane zone autoantibodies, measured by indirect IF microscopy with disease activity: (A), ABSIS score of cutaneous involvement; (D), BPDAl global score $(n=75)$. Relation of titers of circulating IgG anti-BP180 NC16A autoantibodies, measured by ELISA with disease activity: (B), ABSIS score of cutaneous involvement; (E), BPDAl global score $(n=75)$. Relation of titers of circulating lgG anti-BP230 autoantibodies, measured by ELISA with disease activity: (C), ABSIS score of cutaneous involvement; (F), BPDAl global score $(n=75)$. The red line represents the locally weighted scatterplot smoothing line.

$0.31-0.6], p<0.001)$ (Figure 4B), BPDAI global score $(r=0.72$ [95\% CI 0.34-0.73], $p<0.001$ ) (Figure 4E), BPDAI score of total skin involvement ( $r=0.71$ [95\% CI 0.32-0.71], $p<0.001$ ), BPDAI score of blistering activity $(r=0.63$ [95\% CI $0.27-0.69$ ], $p<0.001)$ and the BPDAI score of urticaria/erythema $(r=0.69$ [95\% CI 0.22-0.59], $p<0.001$ ), but not ABSIS objective oral score $(p=0.58)$ and BPDAI mucosal activity score $(p=0.72)$.

We did not observe a significant correlation between the level of circulating anti-BP230 autoantibodies measured by ELISA and the evaluated components of ABSIS and BPDAI scores (Figures 4C,F).

\section{Ex vivo Complement-Activation Capacity of Autoantibodies Correlates With Disease Activity in Patients With Bullous Pemphigoid}

We observed a correlation between the complement-activation capacity of autoantibodies, measured by complement binding test and the ABSIS score of cutaneous involvement ( $r=0.47$ [95\% CI 0.18-0.56], $p<0.001$; Figure 5A), BPDAI global score $(r=$ 0.47 [95\% CI 0.16-0.59], $p<0.001$; Figure 5B), BPDAI score of total skin involvement $(r=0.47$ [95\% CI 0.19-0.61], $p<0.001)$, BPDAI score of blistering activity $(r=0.41$ [95\% CI 0.09-0.62], $p<0.001$; Figure 5C) and BPDAI score of urticaria/erythema $(r=0.45$ [95\% CI 0.15-0.47], $p<0.001$; Figure 5D), but not ABSIS objective oral score $(p=0.8)$ and BPDAI mucosal activity score $(p=0.84)$. Patients with mucosal involvement showed slightly higher values of complement-activating capacity of autoantibodies, compared with patients with only cutaneous involvement (40 [7.5-120] vs. $20[10-40])$, but the results were not statistically significant $(p=0.76)$.

Autoantibodies in patients with positive ELISA findings also showed higher complement activation capacity, compared with patients with negative ELISA findings (40 [10-140] vs. 5 [4.256.25], $p<0.001)$.

Also, patients with positive ELISA values (for BP180-NC16A-, BP230-specific IgG autoantibodies or both) showed statistically significant higher values of ABSIS cutaneous score, BPDAI global score, BPDAI score of erosion activity and BPDAI score of urticaria/erythema, compared with patients with negative ELISA findings (Table 1).

\section{Dermo-Epidermal Separation Measured by Cryosection Assay Shows a Weak Correlation With Disease Activity in Patients With Bullous Pemphigoid}

We observed a weak correlation between the dermo-epidermal separation level, revealed by cryosection assay and the ABSIS score of cutaneous involvement $(r=0.24$ [95\% CI 0.02-0.44], $p=0.04$ ), but not BPDAI global score, BPDAI score of total skin involvement, BPDAI score of blistering activity, BPDAI score of urticaria/erythema, ABSIS oral 1 score and BPDAI score of mucosal activity. We did not observe a statistically significant difference between the level of dermo-epidermal separation, revealed by cryosection assay in the patients with 

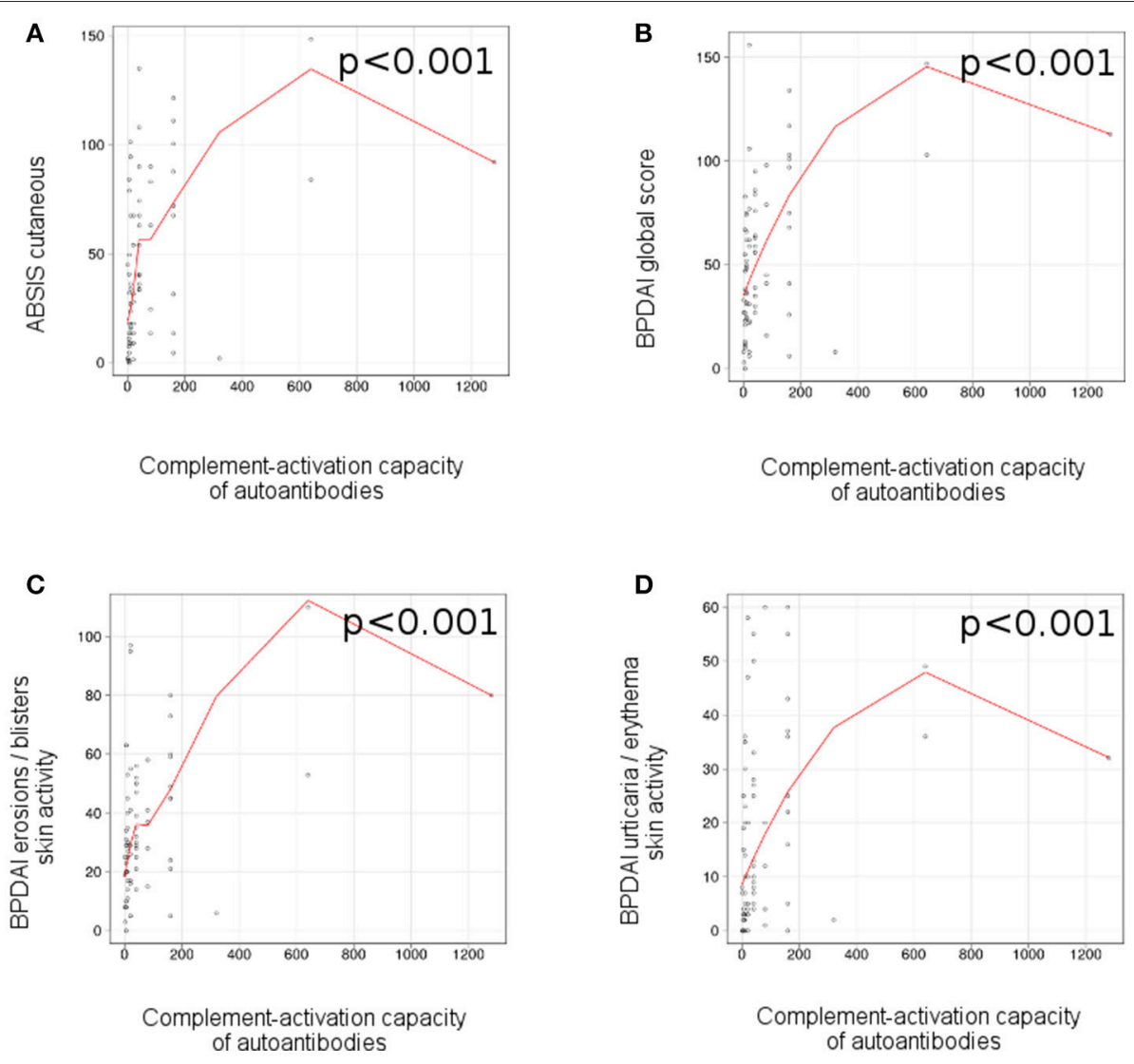

FIGURE 5 | Ex vivo complement-activation capacity of autoantibodies correlates with disease activity in patients with bullous pemphigoid. Relation of ex vivo complement-activation capacity of autoantibodies with disease activity: (A), ABSIS score of cutaneous involvement; (B), BPDAI global score; (C), BPDAl erosions / blisters skin activity; (D), BPDAl urticaria/erythema skin activity $(n=75)$. The red line represents the locally weighted scatterplot smoothing line.

associated mucosal involvement vs. patients with only cutaneous involvement $(p=0.94)$.

\section{High Pemphigoid Disease Activity Associates With Increased Systemic Inflammatory Status}

We did not find a statistically significant correlation between the ESR level taken as a continuous variable $(\mathrm{mm} / \mathrm{h})$ and the immunopathological features, as well as the evaluated components of ABSIS and BPDAI scores.

Patients with an ESR $>30 \mathrm{~mm} / \mathrm{h}$ showed a statistically significant higher level of anti-BP180 NC16A autoantibodies, measured by ELISA, compared to patients with an $\mathrm{ESR}<30$ $\mathrm{mm} / \mathrm{h}$ (174.1 [121.5-323.75] vs. 104.9 [25.25-163.3], $p=0.006)$ (Figure 6A). Also, they showed a higher level of complement activation (40 [20-280] vs. 20 [10-80]), but the results were not statistically significant ( $p=0.06$; Figure 6B).

Patients with an ESR $>30 \mathrm{~mm} / \mathrm{h}$ also showed a statistically significant higher BPDAI global score, compared with patients with an ESR $<30 \mathrm{~mm} / \mathrm{h}$ (64 [55-89] vs. 37.5 [23.75-75.25], $p=$ 0.03; Figure 6C) and a higher BPDAI skin erosion activity score (50 [34-56] vs. $27.5[17-40.5], p=0.005)$.

\section{Immunofluorescence Intensity of C3 Deposits in the Skin of the Patients Does Not Correlate With Complement-Activating Capacity of Pemphigoid Autoantibodies}

We did not find a statistically significant correlation between the complement-activating capacity of autoantibodies, measured by complement binding test and the intensity of complement deposits in the skin, measured by our Image $\mathrm{J}$ algorithm of quantifying fluorescence $(n=27)$, for both Fluorescence difference ( $r=-0.07$ [95\% CI -0.47 to 0.29 ], $p=0.71)$ and Fluorescence ratio ( $r=-0.16$ [95\% CI -0.43 to 0.09 ], $p=0.43$ ).

Also, we did not observe a correlation between the intensity of complement deposits in the skin and the immunopathological features of the patients.

Eighty-seven out of ninety patients showed a positive complement-binding test. The $\mathrm{C} 3$ deposits in DIF were evaluated by Image $\mathrm{J}$ in 27 patients. Out of these, 26 were able to induce complement-fixation ex vivo.

We did not find a statistically significant correlation between the complement-activating capacity of autoantibodies, measured by complement binding test and the intensity of complement deposits in the skin, measured by our Image $\mathrm{J}$ algorithm of 
TABLE 1 | Values of different clinical and immunopathological variables according to ELISA positivity (*).

\begin{tabular}{|c|c|c|c|}
\hline Variable & ELISA positive & ELISA negative & $p$-value \\
\hline Age (years) & $(n=82) 74.5(68-80)$ & $(n=8) 68(60.5-74.25)$ & $0.35 \bullet$ \\
\hline Indirect IF IgG & $1,280(400-2560)$ & $640(560-1600)$ & $0.5 \bullet$ \\
\hline Complement-binding test & $40(10-140)$ & $5(4.25-6.25)$ & $<0.001 \bullet$ \\
\hline Cryosection assay score & $3(3-4)$ & $3(2.75-3)$ & $0.44 \bullet$ \\
\hline ABSIS cutaneous & $(n=68) 40.25(17.62-72.56)$ & $(n=7) 9(5.5-10)$ & $0.01 \bullet$ \\
\hline BPDAl global score & $53.5(30-76.25)$ & $11(8-29)$ & $0.007 \bullet$ \\
\hline BPDAI score of total skin involvement & $49.5(29.25-76.25)$ & $11(8-29)$ & 0.009 \\
\hline BPDAl erosion activity & $30(20-49.25)$ & $8(6.5-26.5)$ & $0.05 \bullet$ \\
\hline BPDAI urticaria/ erythema & $12.5(4-32.25)$ & $2(0-3)$ & $<0.001 \bullet$ \\
\hline Mucosal involvement & Yes $(n=11)$ & No $(n=0)$ & $0.58+$ \\
\hline $\operatorname{ESR}(\mathrm{mm} / \mathrm{h})$ & $(n=66) 18.5(11-32.75)$ & $(n=7) 15(12.5-28)$ & $0.72 \bullet$ \\
\hline
\end{tabular}

-Mann-Whitney $U$ test has been used.

+Fisher Exact test has been used.

${ }^{\star}$ ELISA positivity is defined as presence of a level of anti-BP180 NC16A autoantibodies and/or anti-BP230 autoantibodies $\geq 9 \mathrm{U} / \mathrm{mL}$.
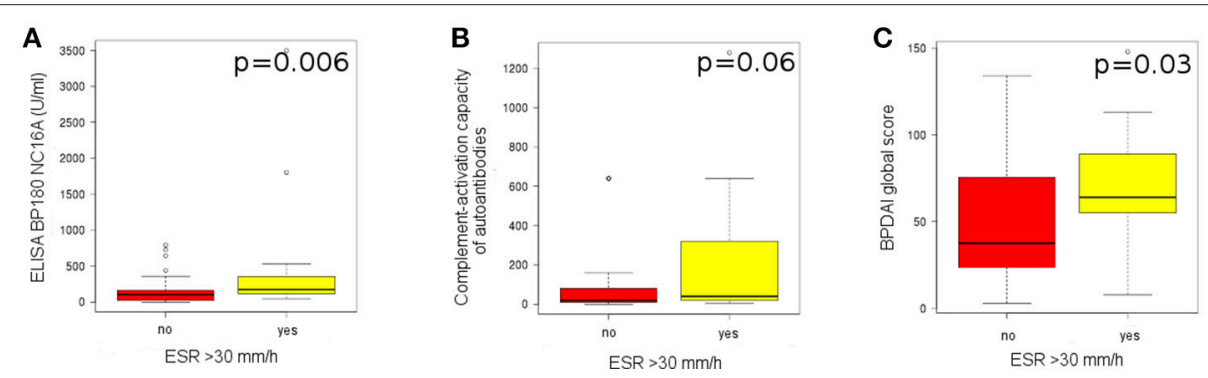

FIGURE 6 | High pemphigoid disease activity associates with increased systemic inflammatory status. (A) Level of anti-BP180 autoantibodies, measured by ELISA, in patients with an ESR $>30 \mathrm{~mm} / \mathrm{h}(n=18)$, compared with patients with an ESR $<30 \mathrm{~mm} / \mathrm{h}(n=55)$. (B) Level of complement activation by lgG autoantibodies, measured by complement-binding test, in patients with an ESR $>30 \mathrm{~mm} / \mathrm{h}(n=18)$, compared with patients with an ESR $<30 \mathrm{~mm} / \mathrm{h}(n=55)$. (C) Level of BPDAl global score, in patients with an ESR $>30 \mathrm{~mm} / \mathrm{h}(n=13)$, compared with patients with an $\mathrm{ESR}<30 \mathrm{~mm} / \mathrm{h}(n=48)$.

quantifying fluorescence in patients with a positive complementbinding test $(n=26)$, for both Fluorescence difference $(r=-0.21$ (95\% CI -0.51 to 0.25$), p=0.315)$ and Fluorescence ratio $(r=$ -0.2 (95\% CI -0.45 to 0.08$), p=0.316$ ).

\section{Immunofluorescence Intensity of C3 Deposits in the Skin of the Patients Does Not Correlate With Disease Activity in Patients With Bullous Pemphigoid}

To investigate the relation between the complement activation in vivo and disease activity, we correlated the intensity of complement deposits in the skin, measured by our Image J algorithm of quantifying fluorescence, for both fluorescence difference and fluorescence ratio with the evaluated components of ABSIS and BPDAI scores $(n=25)$. We did not find a statistically significant correlation between the complement activation in vivo and disease activity.

\section{DISCUSSION}

To investigate the pathogenic relevance of complement activation in pemphigoid patients, we have measured the complement-binding capacity of patients' autoantibodies ex vivo and in the patients' skin and have assessed their correlation with the clinical disease activity as well as their capacity to induce dermal-epidermal separation ex vivo. The salient findings of this study show for the first time that the complement-activating potential of autoantibodies ex vivo correlate well with both their serum levels and with pemphigoid disease activity in patients.

Based on the observation that complement deposits are constantly found, frequently to a higher extent when compared with IgG deposits at the dermal-epidermal junction in patients with bullous and gestational pemphigoid, it was early hypothesized that complement activation plays a major pathogenetic role for skin blistering in pemphigoid diseases $(27,28,41,42)$.

Previous studies have already shown that IgG4 and IgG1 are the predominant IgG subclasses present in the serum and binding to the dermoepidermal junction in patients with bullous pemphigoid. While a NC16A-specific IgG1 response was predominant in the acute phase of bullous pemphigoid, IgG4 was predominantly detected in bullous pemphigoid patients in remission. Using immunoafinity purified IgG subclasses from bullous pemphigoid patients, it has been demonstrated that IgG1, 


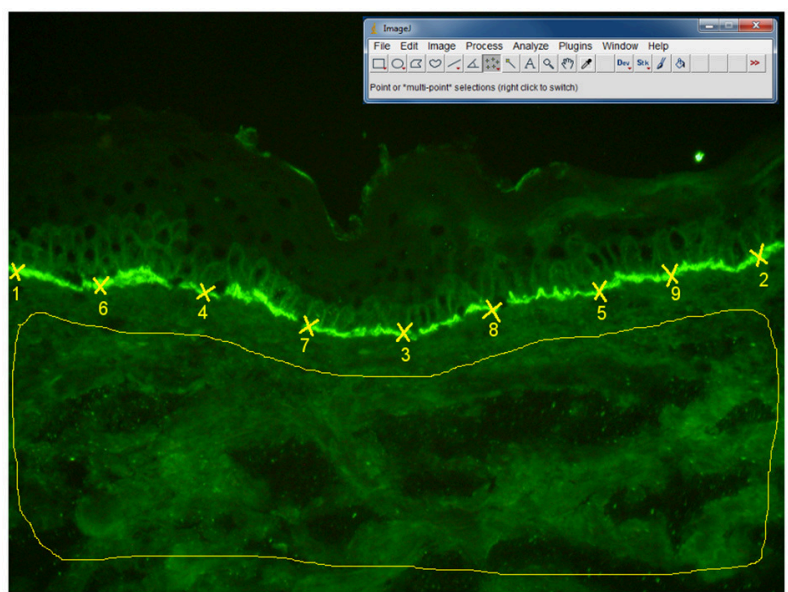

FIGURE 7 | Algorithm of measurement of fluorescence intensity in direct IF microscopy images, in patients with BP ( $n=27$ patients). The fluorescence intensity was assessed by two independent blinded observers using Image $J$ software, $1.47 \mathrm{v}$ version. The fluorescence intensity at the basement membrane was analyzed by selecting 50 points (using Multi-point tool), each point being situated at the half distance between two previous points.

Background fluorescence was evaluated by selecting the whole dermis area, using "Freehand selections" tool. In order to obtain the mean value and standard deviation of the fluorescence intensity for the 50 points on the basement membrane and dermis background we have used the Analyze-Measure-Summarize tool.

but not IgG4 autoantibodies activate the complement system in vitro (24).

Subsequent work using an assay in cryosections of human skin incubated with pemphigoid autoantibodies and granulocytes from healthy donors strengthened this concept by showing that complement inactivation results in less dermal-epidermal separation (33). However, further work using this ex vivo model of autoantibody-induced granulocyte-dependent tissue damage extended and partly corrected these findings. This suggested that, while recruitment of granulocytes heavily depends on complement activation by autoantibodies, none of these 2 events are strictly required for inducing the disease-specific effects in the cryosections, especially when granulocytes at high densities were used $(34,43)$. These findings may be explained by the design of cryosection assay. Since granulocytes in high numbers are directly placed on the sections at the dermal-epidermal junction, they do not have to migrate to the tissue-bound immune complexes. Therefore, chemotactic factors resulting from complement activation are not required for subepidermal split formation (34).

The central finding of our study that the complementactivating capacity of autoantibodies correlates well with the disease activity is in line with observations in animal models of pemphigoid diseases and the pemphigoid-like form of epidermolysis bullosa acquisita induced by the passive transfer of antibodies against the epidermal basement membrane $(14,17,44)$. As expected, complement-activation by autoantibodies showed in general a good correlation with levels of autoantibodies as measured by their indirect IF microscopy titers. Autoantibodies in patients with bullous pemphigoid are mainly targeting the hemidesmosomal proteins collagen XVII/BP180 and BP230 $(45,46)$. Not surprisingly, the best correlation was documented with ELISA levels of BP180 NC16A-specific autoantibodies, which are believed to primarily mediate tissue damage in pemphigoid $(29,47-50)$. Data emerging from another group using mice deficient for murine collagen XVII and expressing the human ortholog of the hemidesmosomal antigen suggest that pemphigoid autoantibodies may induce tissue damage in both complementdependent and complement-independent manner (19, 20, 31). However, the role of complement-independent mechanisms, including antigen depletion for the autoantibody-induced blistering in pemphigoid patients, especially in the generalized, inflammatory disease is still unclear (26). Integrating these novel findings in patients into the existing knowledge framework adds critical support for a pathogenic role of complement activation in the pemphigoid blister formation.

The in vivo deposition of complement at the dermalepidermal junction in patients' skin as measured by the fluorescence intensity of $\mathrm{C} 3$ staining did not correlate with the complement-activating capacity of autoantibodies ex vivo or with the disease scores. The reason for this phenomenon is not known. Beyond issues related to an accurate measurement of tissue-bound complement deposits, one can only speculate that granulocyte recruitment shows a non-linear dependency on the production of complement-derived chemotactic factors. Indeed neutrophil activation in the upper dermis would also result in the production of chemotactic factors such as interleukin-8, which may amplify the recruitment of granulocytes and the extent of skin blistering. These findings parallel previous results suggesting that local complement activation correlates with tissue damage in a non-linear fashion, i.e., it needs to exceed a threshold in order to initiate a pathogenic inflammatory cascade eventually resulting in tissue damage (51).

Skin lesions in pemphigoid patients are usually associated with intense itch, which however has not yet been reliably reproduced in experimental models of pemphigoid diseases. Interestingly, more recent data show that mice carrying a deletion in the NC14A domain of murine type XVII collagen begin scratching early and develop erosions, subepidermal vesicles, eosinophil-rich skin infiltrates, and possibly autoantibodies (52). While not showing full-blown autoimmune blistering disease, these mice may indeed represent a first pemphigoid animal model featuring pruritus in immunocompetent mature animals $(52,53)$. While the pathogenesis of pruritus is complex (54), its mechanisms in pemphigoid diseases are still elusive. However, observations in patients suggest that pemphigoid itch may be related to the presence of IgE autoantibodies against the basement membrane with subsequent eosinophil and mast cell activation $(55,56)$. While a primary pathogenic involvement of complement activation in pruritus in pemphigoid appears unlikely and should be addressed in future studies on mouse models, the correlation of itch as measured by the subjective disease scores with the levels of complement-activation by autoantibodies may be addressed in a prospective future study. 
A further finding of the present study is the correlation between markers of systemic inflammation and complementactivating capacity of autoantibodies. ESR is a robust marker of systemic inflammation, which however does not always correlate with the extend of complement activation as known from findings in patients with systemic lupus erythematosus. Patients with an ESR $>30 \mathrm{~mm} / \mathrm{h}$ showed higher levels of BP180-specific autoantibodies and tendentially an increased level of complement activation by autoantibodies. These findings suggest that increased local inflammation "spills" systemically. Importantly, the present and previous findings show that an ESR $>30 \mathrm{~mm} / \mathrm{h}$ correlates with disease activity and is a risk factor for lethal outcome in patients with pemphigoid (37).

The association of bullous pemphigoid with neurological comorbidities is well characterized (57-59). Even if a major role of autoantibodies in the pathogenesis of neuronal tissue injury in pemphigoid diseases cannot be ruled out at this stage, data regarding an association between presence of neurological comorbidities and level of autoantibodies remains controversial $(60,61)$.

Our present results provide further rationale for the development of complement-targeted therapeutics in pemphigoid and diseases with related complement-dependent pathogenesis. Indeed inhibitors of terminal complement activation such as Eculizumab, which represented a major leap forward in the treatment of paroxysmal nocturnal hemoglobinuria could be repurposed for the treatment of pemphigoid diseases (62). Further complement inhibitors, including those targeting $\mathrm{C} 3$ activation that are currently under development may be also worth considering as therapeutic options in pemphigoid diseases (63).

Measuring the complement-activating autoantibodies may represent a significant improvement over existing diagnostic and monitoring tools in complement-mediated autoimmune diseases. It is known that binding of autoantibodies to their targets per se may not be sufficient to induce disease, which may require triggering Fc-dependent complement activation (64). Measuring specifically levels of complement-fixing autoantibodies by a quantitative complement-mediated assay may thus represent a diagnostic tool of pathogenic relevance, which may be especially useful for monitoring serological activity. In addition to the complement-fixation tests by indirect IF microscopy $(28,65)$, development of a quantitative immunoassay measuring complement activation by antigenspecific autoantibodies should be an important addition to the routine diagnosis of autoimmune diseases.

A limitation of the study is related to the way the evaluation of fluorescence intensity of $\mathrm{C} 3$ in direct fluorescence sections correlates with complement-activating capacity of autoantibodies.

An issue to be addressed in future studies is represented by the role of the eosinophils in the pathogenesis of bullous pemphigoid and possibly in complement activation. In a recent published study, it was shown that IL-5-activated eosinophils directly contribute to blister formation in the presence of bullous pemphigoid autoantibodies, in an ex vivo human disease model (66). In conclusion, the results of our study show that autoantibody complement-binding capacity correlates with the extent of the blistering disease, strongly suggesting a pathogenic role of complement activation in pemphigoid patients. These findings should greatly facilitate the development of more specific diagnostic and monitoring tools as well as the design of complement-targeted therapeutic approaches in autoimmune inflammatory diseases.

\section{CONCLUSIONS}

In conclusion, complement activation by autoantibodies ex vivo as measured by the complement-fixation assay in serum was correlated with the clinical disease activity in patients with bullous pemphigoid. Our study provides for the first time evidence in patients for a pathogenic role of complement activation in bullous pemphigoid and should greatly facilitate the development of novel diagnostic tools and of more specific therapies for complement-dependent autoimmune injury.

\section{DATA AVAILABILITY STATEMENT}

The datasets during and/or analyzed during the current study are available from the corresponding author on reasonable request.

\section{AUTHOR CONTRIBUTIONS}

$\mathrm{RC}$ and CS conceived of the study, made the design, coordinated the study, and drafted the manuscript. $A B$ provided all patient data and biological samples. VF and RC collected the epidemiological data. RC, AL, and MM performed the laboratory experiments and interpretation if data. D-CL performed the statistical analysis. D-CL, RC, AL, and MM performed interpretation of the results. MH contributed to drafting and critical revision of the manuscript. All authors read and approved the final manuscript.

\section{FUNDING}

The article processing charge was funded by the German Research Foundation (DFG) and the Albert Ludwigs University Freiburg in the funding programme Open Access Publishing. This work was supported by grants from the Deutsche Forschungsgemeinschaft SI-1281/5-1 and SI-1281/6-1 (CS) and HE-1602/13-1 (MH). D-CL is a fellow of POSDRU grant no. 159/1.5/S/138776 Model colaborativ institutional pentru translatarea cercetarii stiintifice biomedicale in practica clinicaTRANSCENT.

\section{ACKNOWLEDGMENTS}

The authors acknowledge Mrs. Dorina Ciuce, Mrs. Ofelia Vladau and Mrs. Garofita Gadalean, from the Department of Dermatology, Iuliu Hatieganu University of Medicine and Pharmacy Cluj-Napoca, for their assistance in performance of the direct IF microscopy and ELISA. 


\section{REFERENCES}

1. Goodnow CC, Vinuesa CG, Randall KL, Mackay F, Brink R. Control systems and decision making for antibody production. Nat Immunol. (2010) 11:681-8. doi: 10.1038/ni.1900

2. Berrih-Aknin S, Le Panse R. Myasthenia gravis: a comprehensive review of immune dysregulation and etiological mechanisms. J Autoimmun. (2014) 52:90-100. doi: 10.1016/j.jaut.2013.12.011

3. Davidson A, Diamond B. Autoimmune diseases. N Engl J Med. (2001) 45:34050. doi: 10.1056/NEJM200108023450506

4. Otten JV, Hashimoto T, Hertl M, Payne AS, Sitaru C. Molecular diagnosis in autoimmune skin blistering conditions. Curr Mol Med. (2014) 14:69-95. doi: $10.2174 / 15665240113136660079$

5. Hudson BG, Tryggvason K, Sundaramoorthy M, Neilson EG. Alport's syndrome, goodpasture's syndrome, and type IV collagen. $N$ Engl J Med. (2003) 348:2543-56. doi: 10.1056/NEJMra022296

6. Ludwig RJ, Vanhoorelbeke K, Leypoldt F, Kaya Z, Bieber K, McLachlan SM, et al. Mechanisms of autoantibody-induced pathology. Front Immunol. (2017) 8:603. doi: 10.3389/fimmu.2017.00603

7. Hammers CM, Stanley JR. Mechanisms of disease: pemphigus and bullous pemphigoid. Annu Rev Pathol. (2016) 11:175-97. doi: 10.1146/annurev-pathol-012615-044313

8. Liu Y, Li L, Xia Y. BP180 Is Critical in the autoimmunity of bullous pemphigoid. Front Immunol. (2017) 8:1752. doi: 10.3389/fimmu.2017. 01752

9. Faurschou M, Jayne DRW. Anti-B cell antibody therapies for inflammatory rheumatic diseases. Ann Rev Med. (2014) 65:263-78. doi: 10.1146/annurev-med-070912-133235

10. Sitaru C, Zillikens D. Mechanisms of blister induction by autoantibodies. Exp Dermatol. (2005) 14:861-75. doi: 10.1111/j.1600-0625.2005.00367.x

11. Sturfelt G, Truedsson L. Complement in the immunopathogenesis of rheumatic disease. Nat Rev Rheumatol. (2012) 8:458-68. doi: 10.1038/nrrheum.2012.75

12. Panelius J, Meri S. Complement system in dermatological diseases - fire under the skin. Front Med. (2015) 2:3. doi: 10.3389/fmed.2015.00003

13. Lennon VA, Seybold ME, Lindstrom JM, Cochrane C, Ulevitch R. Role of complement in the pathogenesis of experimental autoimmune myasthenia gravis. J Exp Med. (1978) 147:973-83. doi: 10.1084/jem.147.4.973

14. Liu Z, Giudice GJ, Swartz SJ, Fairley JA, Till GO, Troy JL, et al. The role of complement in experimental bullous pemphigoid. J Clin Invest. (1995) 95:1539-44. doi: 10.1111/j.1749-6632.2012.06783.x

15. Ji H, Ohmura K, Mahmood U, Lee DM, Hofhuis FMA, Boackle SA, et al. Arthritis critically dependent on innate immune system players. Immunity (2002) 16:157-68. doi: 10.1016/S1074-7613(02)00275-3

16. Lazarova Z, Yee C, Darling T, Briggaman RA, Yancey KB. Passive transfer of anti-laminin 5 antibodies induces subepidermal blisters in neonatal mice. $J$ Clin Invest. (1996) 98:1509-18. doi: 10.1172/JCI118942

17. Sitaru C, Mihai S, Otto C, Chiriac MT, Hausser I, Dotterweich B, et al. Induction of dermal-epidermal separation in mice by passive transfer of antibodies specific to type VII collagen. J Clin Invest. (2005) 115:870-8. doi: 10.1172/JCI200521386

18. Yamamoto K, Inoue N, Masuda R, Fujimori A, Saito T, Imajoh-Ohmi S, et al. Cloning of hamster type XVII collagen cDNA, and pathogenesis of anti-type XVII collagen antibody and complement in hamster bullous pemphigoid. J Invest Dermatol. (2002) 118:485-92. doi: 10.1046/j.0022-202x.2001.01 683. $\mathrm{x}$

19. Natsuga K, Nishie W, Shinkuma S, Ujiie H, Nishimura M, Sawamura D, et al. Antibodies to pathogenic epitopes on type XVII collagen cause skin fragility in a complement-dependent and -independent manner. J Immunol. (2012) 188:5792-9. doi: 10.4049/jimmunol.1003402

20. Ujiie H, Sasaoka T, Izumi K, Nishie W, Shinkuma S, Natsuga K, et al. Bullous pemphigoid autoantibodies directly induce blister formation without complement activation. J Immunol. (2014) 193:4415-28. doi: 10.4049/jimmunol.1400095

21. Mihai S, Hirose M, Wang Y, Thurman JM, Holers VM, Morgan BP, et al. Specific inhibition of complement activation significantly ameliorates autoimmune blistering disease in mice. Front Immunol. (2018) 9:535. doi: 10.3389/fimmu.2018.00535
22. Anhalt GJ, Till GO, Diaz LA, Labib RS, Patel HP, Eaglstein NF. Defining the role of complement in experimental pemphigus vulgaris in mice. J Immunol. (1986) 137:2835-40.

23. Florea F, Koch M, Hashimoto T, Sitaru C. Autoimmunity against laminins. Clin Immunol. (2016) 170:39-52. doi: 10.1016/j.clim.2016.07.021

24. Sitaru C, Mihai S, Zillikens D. The relevance of the IgG subclass of autoantibodies for blister induction in autoimmune bullous skin diseases. Arch Dermatol Res. (2007) 299:1-8. doi: 10.1007/s00403-007-0734-0

25. Karsten CM, Beckmann T, Holtsche MM, Tillmann J, Tofern S, Schulze FS, et al. Tissue destruction in bullous pemphigoid can be complement independent and may be mitigated by C5aR2. Front Immunol. (2018) 9:488. doi: $10.3389 /$ fimmu.2018.00488

26. Sitaru C. Bullous pemphigoid: a prototypical antibody-mediated organspecific autoimmune disease. J Invest Dermatol. (2009) 129:822-4. doi: $10.1038 /$ jid.2009.12

27. Jordon RE, Day NK, Sams WM, Good RA. The complement system in bullous pemphigoid. I. Complement and component levels in sera and blister fluids. $J$ Clin Invest. (1973) 52:1207-14. doi: 10.1172/JCI107288

28. Jordon RE, Heine KG, Tappeiner G, Bushkell LL, Provost TT. The immunopathology of herpes gestationis. Immunofluorescence studies and characterization of "HG factor." J Clin Invest. (1976) 57:1426-31. doi: 10.1172/JCI108412

29. Liu Z, Diaz LA, Troy JL, Taylor AF, Emery DJ, Fairley JA, et al. A passive transfer model of the organ-specific autoimmune disease, bullous pemphigoid, using antibodies generated against the hemidesmosomal antigen, BP180. J Clin Invest. (1993) 92:2480-8. doi: 10.1172/JCI116856

30. Nelson KC, Zhao M, Schroeder PR, Li N, Wetsel RA, Diaz LA, et al. Role of different pathways of the complement cascade in experimental bullous pemphigoid. J Clin Invest. (2006) 116:2892-900. doi: 10.1172/JCI17891

31. Li Q, Ujiie H, Shibaki A, Wang G, Moriuchi R, Qiao H, et al. Human IgG1 monoclonal antibody against human collagen 17 noncollagenous 16A domain induces blisters via complement activation in experimental bullous pemphigoid model. J Immunol. (2010) 185:7746-55. doi: 10.4049/jimmunol.1000667

32. Iwata H, Kamio N, Aoyama Y, Yamamoto Y, Hirako Y, Owaribe K, et al. IgG from patients with bullous pemphigoid depletes cultured keratinocytes of the $180-\mathrm{kDa}$ bullous pemphigoid antigen (Type XVII collagen) and weakens cell attachment. J Invest Dermatol. (2009) 129:919-26. doi: 10.1038/jid.2008.305

33. Gammon WR, Merritt CC, Lewis DM, Sams WM, Carlo JR, Wheeler CE. An in vitro model of immune complex-mediated basement membrane zone separation caused by pemphigoid antibodies, leukocytes, and complement. $J$ Invest Dermatol. (1982) 78:285-90. doi: 10.1111/1523-1747.ep12507222

34. Sitaru C, Schmidt E, Petermann S, Munteanu LS, Bröcker E-B, Zillikens D. Autoantibodies to bullous pemphigoid antigen 180 induce dermal-epidermal separation in cryosections of human skin. J Invest Dermatol. (2002) 118:66471. doi: 10.1046/j.1523-1747.2002.01720.x

35. Pfütze $M$, Niedermeier A, Hertl $M$, Eming R. Introducing a novel Autoimmune Bullous Skin Disorder Intensity Score (ABSIS) in pemphigus. Eur J Dermatol. (2007) 17:4-11. doi: 10.1684/ejd.2007.0090

36. Murrell DF, Daniel BS, Joly P, Borradori L, Amagai M, Hashimoto $\mathrm{T}$, et al. Definitions and outcome measures for bullous pemphigoid: recommendations by an international panel of experts. J Am Acad Dermatol. (2012) 66:479-85. doi: 10.1016/j.jaad.2011.06.032

37. Rzany B, Partscht K, Jung M, Kippes W, Mecking D, Baima B, et al. Risk factors for lethal outcome in patients with bullous pemphigoid: low serum albumin level, high dosage of glucocorticosteroids, and old age. Arch Dermatol. (2002) 138:903-8. doi: 10.1001/archderm.138.7.903

38. Kobayashi M, Amagai M, Kuroda-Kinoshita K, Hashimoto T, Shirakata Y, Hashimoto K, et al. BP180 ELISA using bacterial recombinant NC16a protein as a diagnostic and monitoring tool for bullous pemphigoid. J Dermatol Sci. (2002) 30:224-32. doi: 10.1016/S0923-1811(02)00109-3

39. Yoshida M, Hamada T, Amagai M, Hashimoto K, Uehara R, Yamaguchi $\mathrm{K}$, et al. Enzyme-linked immunosorbent assay using bacterial recombinant proteins of human BP230 as a diagnostic tool for bullous pemphigoid. J Dermatol Sci. (2006) 41:21-30. doi: 10.1016/j.jdermsci.2005.11.002

40. R Development Core Team. $R$ : A Language And Environment for Statistical Computing. Vienna: R Foundation for Statistical Computing. (2010). Available online at: http://www.R-project.org (Accessed 19 December, 2014). 
41. Jordon RE. Complement activation in bullous skin diseases. J Invest Dermatol. (1975) 65:162-9. doi: 10.1111/1523-1747.ep12598113

42. Jordon RE, Kawana S, Fritz KA. Immunopathologic mechanisms in pemphigus and bullous pemphigoid. I Invest Dermatol. (1985) 85(1 Suppl.):72s-8s. doi: 10.1111/1523-1747.ep12275497

43. Gammon WR, Inman AO, Wheeler CE. Differences in complementdependent chemotactic activity generated by bullous pemphigoid and epidermolysis bullosa acquisita immune complexes: demonstration by leukocytic attachment and organ culture methods. J Invest Dermatol. (1984) 83:57-61. doi: 10.1111/1523-1747.ep12261694

44. Liu Z, Sui W, Zhao M, Li Z, Li N, Thresher R, et al. Subepidermal blistering induced by human autoantibodies to BP180 requires innate immune players in a humanized bullous pemphigoid mouse model. J Autoimmun. (2008) 31:331-8. doi: 10.1016/j.jaut.2008.08.009

45. Thoma-Uszynski S, Uter W, Schwietzke S, Hofmann SC, Hunziker T, Bernard P, et al. BP230- and BP180-specific auto-antibodies in bullous pemphigoid. $J$ Invest Dermatol. (2004) 122:1413-22. doi: 10.1111/j.0022-202X.2004.22603.x

46. Di Zenzo G, Thoma-Uszynski S, Fontao L, Calabresi V, Hofmann SC, Hellmark T, et al. Multicenter prospective study of the humoral autoimmune response in bullous pemphigoid. Clin Immunol. (2008) 128:41526. doi: 10.1016/j.clim.2008.04.012

47. Nishie W, Sawamura D, Goto M, Ito K, Shibaki A, McMillan JR, et al. Humanization of autoantigen. Nat Med. (2007) 13:378-83. doi: $10.1038 / \mathrm{nm} 1496$

48. Oswald E, Sesarman A, Franzke C-W, Wölfle U, Bruckner-Tuderman L, Jakob $\mathrm{T}$, et al. The flavonoid luteolin inhibits Fc $\gamma$-dependent respiratory burst in granulocytes, but not skin blistering in a new model of pemphigoid in adult mice. PLoS ONE (2012) 7:e31066. doi: 10.1371/journal.pone.0031066

49. Chiriac MT, Licarete E, Sas AG, Rados AM, Lupan I, Chiriac AM, et al. Passive transfer of collagen XVII-specific antibodies induces sustained blistering disease in adult mice. Orphanet J Rare Dis. (2013) 8:17. doi: 10.1186/1750-1172-8-17

50. Hofmann S, Thoma-Uszynski S, Hunziker T, Bernard P, Koebnick C, Stauber A, et al. Severity and phenotype of bullous pemphigoid relate to autoantibody profile against the $\mathrm{NH} 2$ - and $\mathrm{COOH}$-terminal regions of the BP180 ectodomain. J Invest Dermatol. (2002) 119:1065-73. doi: 10.1046/j.1523-1747.2002.19529.x

51. Sesarman A, Sitaru AG, Olaru F, Zillikens D, Sitaru C. Neonatal Fc receptor deficiency protects from tissue injury in experimental epidermolysis bullosa acquisita. J Mol Med. (2008) 86:951-9. doi: 10.1007/s00109-008-0366-7

52. Hurskainen T, Kokkonen N, Sormunen R, Jackow J, Löffek S, Soininen R, et al. Deletion of the major bullous pemphigoid epitope region of collagen XVII induces blistering, autoimmunization, and itching in mice. J Invest Dermatol. (2015) 135:1303-10. doi: 10.1038/jid.2014.443

53. Yancey KB. Itch, eosinophils, and autoimmunity: a novel murine model of bullous pemphigoid. J Invest Dermatol. (2015) 135:1213-5. doi: $10.1038 /$ jid.2014.537

54. Han L, Dong X. Itch mechanisms and circuits. Annu Rev Biophys. (2014) 43:331-55. doi: 10.1146/annurev-biophys-051013-022826

55. Fairley JA, Baum CL, Brandt DS, Messingham KAN. Pathogenicity of IgE in autoimmunity: successful treatment of bullous pemphigoid with omalizumab.
J Allergy Clin Immunol. (2009) 123:704-5. doi: 10.1016/j.jaci.2008. 11.035

56. Simon D, Hoesli S, Roth N, Staedler S, Yousefi S, Simon H-U. Eosinophil extracellular DNA traps in skin diseases. J Allergy Clin Immunol. (2011) 127:194-9. doi: 10.1016/j.jaci.2010.11.002

57. Bastuji-Garin S, Joly P, Lemordant P, Sparsa A, Bedane C, Delaporte E, et al. Risk factors for bullous pemphigoid in the elderly: a prospective case-control study. J Invest Dermatol. (2011) 131:637-43. doi: 10.1038/jid.20 10.301

58. Langan SM, Groves RW, West J. The relationship between neurological disease and bullous pemphigoid: a population-based case-control study. $J$ Invest Dermatol. (2011) 131:631-6. doi: 10.1038/jid.2010.357

59. Chen YJ, Wu CY, Lin MW, Chen TJ, Liao KK, Chen YC, et al. Comorbidity profiles among patients with bullous pemphigoid: a nationwide population-based study. Br J Dermatol. (2011) 165:593-9. doi: 10.1111/j.1365-2133.2011.10386.x

60. Taghipour K, Chi C-C, Bhogal B, Groves RW, Venning V, Wojnarowska F. Immunopathological characteristics of patients with bullous pemphigoid and neurological disease. J Eur Acad Dermatol Venereol. (2014) 28:569-73. doi: $10.1111 /$ jdv.12136

61. Gambichler T, Segert H, Höxtermann S, Schmitz L, Altmeyer P, Teegen B. Neurological disorders in patients with bullous pemphigoid: clinical and experimental investigations. J Eur Acad Dermatol Venereol. (2015) 29:175862. doi: $10.1111 /$ jdv. 12995

62. Risitano AM. Current and future pharmacologic complement inhibitors. Hematol Oncol Clin North Am. (2015) 29:561-82. doi: 10.1016/j.hoc.2015.01.009

63. Thurman JM. Therapeutic regulation of complement in patients with renal disease - where is the promise? Clin Nephrol. (2012) 77:413-23. doi: $10.5414 / \mathrm{CN} 107220$

64. Sitaru C, Chiriac MT, Mihai S, Büning J, Gebert A, Ishiko A, et al. Induction of complement-fixing autoantibodies against type VII collagen results in subepidermal blistering in mice. J Immunol. (2006) 177:3461-8.

65. Katz SI, Hertz KC, Yaoita H. Herpes gestationis. Immunopathology and characterization of the HG factor. J Clin Invest. (1976) 57:1434-41. doi: $10.1172 /$ JCI108413

66. De Graauw E, Sitaru C, Horn M, Borradori L, Yousefi S, Simon H-U, et al. Evidence for a role of eosinophils in blister formation in bullous pemphigoid. Allergy (2017) 72:1105-13. doi: 10.1111/all.13131

Conflict of Interest Statement: The authors declare that the research was conducted in the absence of any commercial or financial relationships that could be construed as a potential conflict of interest.

Copyright (๑) 2018 Chiorean, Baican, Mustafa, Lischka, Leucuta, Feldrihan, Hertl and Sitaru. This is an open-access article distributed under the terms of the Creative Commons Attribution License (CC BY). The use, distribution or reproduction in other forums is permitted, provided the original author(s) and the copyright owner(s) are credited and that the original publication in this journal is cited, in accordance with accepted academic practice. No use, distribution or reproduction is permitted which does not comply with these terms. 